\title{
OPEN Measuring spontaneous and automatic processing of magnitude and parity information of Arabic digits by frequency-tagging EEG
}

\begin{abstract}
Mathieu Guillaume ${ }^{1,3 凶}$, Alexandre Poncin ${ }^{2,3}$, Christine Schiltz ${ }^{2,4}$ \& Amandine Van Rinsveld ${ }^{1,4}$
Arabic digits (1-9) are everywhere in our daily lives. These symbols convey various semantic information, and numerate adults can easily extract from them several numerical features such as magnitude and parity. Nonetheless, since most studies used active processing tasks to assess these properties, it remains unclear whether and to what degree the access to magnitude and especially to parity is automatic. Here we investigated with EEG whether spontaneous processing of magnitude or parity can be recorded in a frequency-tagging approach, in which participants are passively stimulated by fast visual sequences of Arabic digits. We assessed automatic magnitude processing by presenting a stream of frequent small digit numbers mixed with deviant large digits (and the reverse) with a sinusoidal contrast modulation at the frequency of $10 \mathrm{~Hz}$. We used the same paradigm to investigate numerical parity processing, contrasting odd digits to even digits. We found significant brain responses at the frequency of the fluctuating change and its harmonics, recorded on electrodes encompassing right occipitoparietal regions, in both conditions. Our findings indicate that both magnitude and parity are spontaneously and unintentionally extracted from Arabic digits, which supports that they are salient semantic features deeply associated to digit symbols in long-term memory.
\end{abstract}

Number symbols convey a large range of different semantic properties, such as being a prime number, being a multiple of another number, or belonging to the power of another number. While these various properties need to be assimilated by long and laborious cultural learning processes, some of them are critical for understanding number symbols' essence ${ }^{1}$. Among these properties, cardinality (or numerosity) and parity are of crucial interest because they are both acquired at an early stage of numeracy learning and they are known to increasingly become salient properties of number symbols ${ }^{2-7}$. The question whether and to what extent such semantic number properties are automatically activated has occupied the numerical cognition literature since several decades ${ }^{8-22}$. This issue is essential because it directly questions the nature of semantic representation of numbers, as well as the developmental trajectory of numerical and mathematical learning ${ }^{23-29}$. For instance, 3 rd graders typically associate digits based on magnitude characteristics when they are asked to categorize numbers according to similarity, while 6th graders are more influenced by parity; adults, for their part, equally categorize digits on their parity and magnitude features ${ }^{30}$.

\section{Automaticity in magnitude processing}

Automaticity is usually defined as an unconscious and unintentional process, that might occur in parallel with other processes ${ }^{31}$, and that happens without monitoring ${ }^{32,33}$. From a general cognitive perspective, automatic (and intuitive) processes are generally contrasted to slow logical reasoning processes ${ }^{34-37}$. Despite this duality, it has been argued that a clearer distinction should be made within automatic processes, between two different

\footnotetext{
${ }^{1}$ Center for Research in Cognitive Neuroscience $(C R C N)$, Université Libre de Bruxelles, 50, Avenue Franklin Roosevelt, CP 191, 1050 Brussels, Belgium. ${ }^{2}$ Cognitive Science and Assessment Institute (COSA), University of Luxembourg, Esch-sur-Alzette, Luxembourg. ${ }^{3}$ These authors contributed equally: Mathieu Guillaume and Alexandre Poncin. ${ }^{4}$ These authors jointly supervised this work: Christine Schiltz and Amandine Van Rinsveld. ${ }^{\varpi}$ email: maguilla.ulb@gmail.com
} 
types of automatic processes ${ }^{21,32,38}$. The first automatic process type is intentional, used when it is necessary and beneficial for the ongoing task, for instance manipulating a number during calculation or reading words in a sentence. The second type is autonomous, unintentional and used even when its processing is irrelevant to the ongoing task. It is noteworthy that pointing out unintentional automatic processing from intentional processes is experimentally complicated. It is actually not possible to disentangle a useful and intentional process from an autonomous process when this process is required for the task ${ }^{21}$.

Despite of these difficulties, three effects could depict automatic processing with number magnitude. The first is the SNARC effect (Spatial Numerical Association of Response Codes) which is characterized by an influence of the magnitude while participants are asked to categorize numbers according to their parity ${ }^{38}$. In particular, participants are faster to categorize small numbers with their left hand and large numbers with their right hand independently of the number's parity. Many authors explain this effect by suggesting the automatic activation of a mental number line ${ }^{39}$ during the task, where the smaller numbers are mapped on the left and the larger numbers on the right (for alternative mechanisms underlying the SNARC effect, please also consider the dual route mode $^{40,41}$, the polarity correspondence account ${ }^{42}$, and the working memory account ${ }^{43-48}$ ). Nonetheless, because parity is also a numerical dimension, experimental settings could trigger the automatic processing of magnitude. The triggering could happen when the relevant dimension (i.e., parity in SNARC effect) and the irrelevant dimension (i.e., magnitude in SNARC effect) are related. In other words, parity and magnitude are both numeric, thus it is difficult to know if magnitude activation observed during the SNARC effect is an autonomous or an intentional automatic processing ${ }^{21}$. Note that some SNARC studies use tasks not entailing any numerical triggering such as orientation discrimination ${ }^{49}$, plural versus singular nouns ${ }^{50}$, line bisection ${ }^{51}$ or colour discrimination ${ }^{52,53}$; significant SNARC effects reported in these studies could therefore be taken as convincing marks of automaticity of numerical magnitude processing.

A second domain revealing automatic number magnitude processing refers to the interference effects observed in tasks similar to the well-known Stroop experiment ${ }^{54}$. One type of such numerical interference effects is for instance the semantic congruity effect. Adults are indeed faster to judge the physical size of two numbers when the bigger number was also the larger in terms of numerical magnitude (e.g., comparing $\mathbf{5}$ vs. 2 is easier than 5 vs. 2) $)^{13,22,25,55,56}$. Another example is the size Congruency Effect happening when participants are asked to choose between two displays with digits, the display with the more numerous array of digits. Participants are faster during this task when the display comprising more digits also contains numerically larger digits and the screen with fewer digits also contains numerically smaller digits ${ }^{18}$. However, these interference effects are only observed in numerical tasks and consequently numerical magnitude could be triggered by the task itself ${ }^{21}$. According to this line of reasoning, interference effects are thus not sufficient to argue that number magnitude is accessed automatically in an autonomous manner.

Third, there is the distance effect, which refers to the fact that comparing close numbers (e.g., 4 and 6 ) is harder than comparing more distant numbers ( 2 and 8$)$. In other words, the difficulty of comparing two numbers is inversely related to the numerical distance between these two numbers ${ }^{57}$. Distance effects are found when participants are explicitly instructed to compare numbers ${ }^{11}$, but also when participants had simply to say if two Arabic numbers were the same or not ${ }^{13}$. Nonetheless, such evidence has also been assumed to be insufficient to accept the distance effect as a proof of autonomous automatic processing ${ }^{21}$. That is to say, even if the distance effect occurs in non-numerical tasks, processing the magnitude in this kind of tasks would be beneficial for the task performance. Distance effects could therefore be a marker of intentional automatic processing rather than marker of autonomous automatic processing.

\section{Automaticity in parity processing}

Regarding parity, a number can only belong to one out of two categories: 'even' or 'odd', indicating that a number is divisible by two or not. Parity judgments are typically learned relatively early, around the two first years of formal education in western cultures ${ }^{53}$, and even earlier in eastern cultures ${ }^{58}$. While some authors argued that parity is computed by dividing a number by two ${ }^{59}$, others proposed that parity information is stored as declarative memory content ${ }^{60,61}$. According to the latter, parity access could not be the result of computations because there is no size effect in parity tasks. More precisely, if the determination of parity were up to dividing by two, small numbers should be processed faster than large numbers, which is not the case. In the same vein, certain digits with specific properties are categorized faster as odd or even ${ }^{4}$. This is for instance the case for numbers that are a power of two (i.e., 2, 4 and 8) and for prime numbers (i.e., 3, 5 and 7). In particular, the more specificities a given number has, the faster their parity will be accessed ${ }^{60}$. These findings are in line with a recent study proposing that the objective, mathematically defined parity differs to a certain degree from the "perceived" parity, as some numbers seem to be more prototypical for their categories ${ }^{62}$.

To the best of our knowledge, there are only a limited number of investigations concerning the automaticity of parity processing. A study of parity categorization using subliminal priming of Arabic numbers (i.e., participants could not consciously see the primed digits) before the onset of a given target number word showed that participants were faster to categorize the target when the primed digit and the target number had the same parity $^{63}$. These results are in favour of an automatic access to the parity information of digits. This is in line with other studies that assessed arithmetical fact verifications based on the parity of the multipliers or addends ${ }^{64}$. In particular, those tasks consisted in judging the correctness of additions or multiplications facts. If the parity rules (such as: addition or multiplication of two even numbers gives an even number) were not respected, participants were faster to say that arithmetical facts were false ${ }^{61}$. Some authors argued that the application of the parity rules is unconscious and automatic because some of their participants reported that they were unaware of using the parity rules, while their results indicated its utilisation ${ }^{64}$. Nevertheless, in both previous paradigms, the experimental task required direct manipulation of the number symbol and therefore can only point towards 
a

Magnitude condition - 1, 2, 3, 4 vs. 6, 7, 8,9

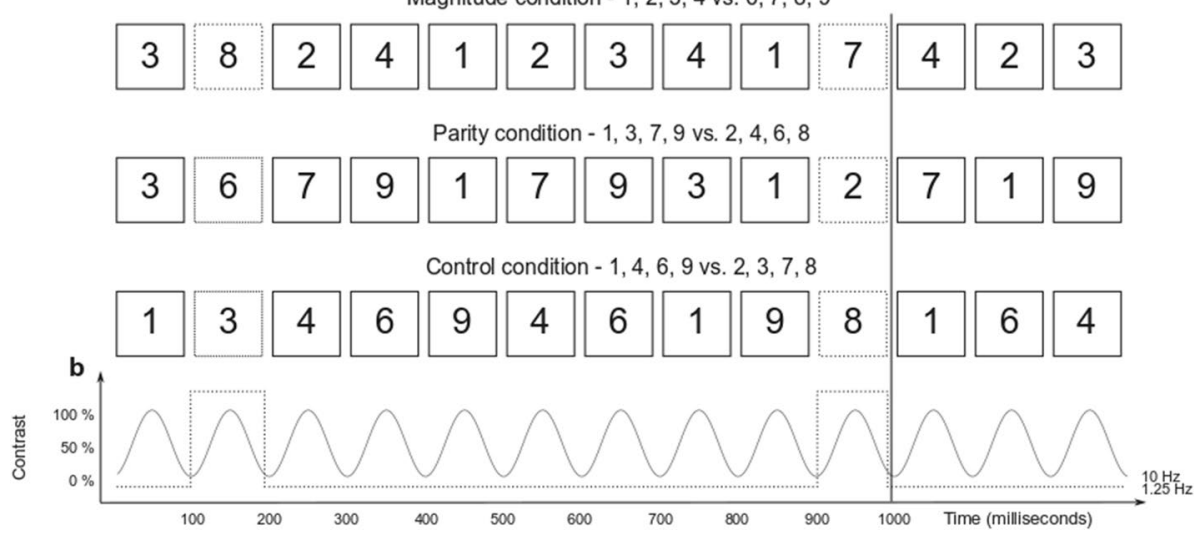

Figure 1. Illustration of the Fast Periodic Visual Stimulation paradigm. (a) During four $60 \mathrm{~s}$ sessions, Arabic digits between 1 and 9 (except 5) were periodically displayed at the base frequency of $10 \mathrm{~Hz}$. In all conditions, digits from a determined standard category were periodically displayed at the base frequency, while digits from another deviant category were displayed at the oddball frequency of $1.25 \mathrm{~Hz}$. In the Magnitude condition, categories were based on the magnitude of the digits (smaller or larger than five). In the Parity condition, categories were constructed as a function of the parity of the Arabic digit (odd or even). In the Control condition, categories were arbitrary. Fonts, font sizes, and positions are here constant for better readability. (b) Illustration of the onset and the offset of each stimulus following the sinusoidal contrast modulation from a 0 to $100 \%$ contrast.

intentional automatic processing ${ }^{21}$, leaving open the question whether autonomous unintentional automatic parity processing might also arise in some circumstances.

\section{Neural correlates of (automatic) number processing}

Neuroimaging studies consistently indicated that the parietal cortex and more specifically the intra-parietal sulcus (IPS) plays a pivotal role in the processing of numerical information ${ }^{65-69}$. Indeed, this region has been associated to a various range of tasks involving the manipulation of numerical material ${ }^{70,71}$. Magnitude comparison of number symbols activate more predominantly the right IPS, for conjoint symbolic and non-symbolic comparison $^{72}$, but also for exclusively symbolic comparison ${ }^{68,73}$. Moreover, the same bilateral IPS region was activated during numerical magnitude comparison as well as parity categorization in a PET study ${ }^{67}$, in which the authors consequently proposed that parity and magnitude are processed in neighbouring areas. It was also proposed that both the occipitoparietal network used for perceptual and representational processing of Arabic digits and the frontoparietal network used for semantic processing are involved in numerical processing such as magnitude comparisons and arithmetical facts retrieval ${ }^{68,69}$.

The parietal cortex is also associated with automatic processing of number symbol semantics. Tasks requiring the simple detection of visual numerals or number words activate the IPS significantly more than the same tasks with non-numerical stimuli (e.g., detection of letters or colours ${ }^{74}$ ). To the best of our knowledge, only a few studies further investigated automatic responses to number symbol magnitudes, and none examined automatic parity processing. Noticeably, using fMRI, a study reported stronger BOLD responses for large digits (8 or 9) than for small digits ( 1 or 2 ) within two bilateral parietal regions (i.e., the inferior parietal lobule and the IPS), when investigating the neural correlates of non-predictive number cues ${ }^{75}$. Other studies reported greater ERP amplitudes across parietal electrode regions for small digits compared to large digits, in a task where numbers were used as non-informative attentional cues ${ }^{76,77}$. Nonetheless, imaging evidence in favour of unintentional processing of magnitude or parity is currently lacking.

\section{Current study and experimental design}

In the present study, we aim at assessing the autonomous unintentional automaticity of magnitude and parity processing, carefully avoiding any triggering of numerical information processing (such that the existence of autonomous unintentional automaticity can be probed $^{21}$ ). For that purpose, we used a sensitive frequency-tagging approach - Fast Periodic Visual Stimulation (FPVS) - to record electrophysiological responses tagged at the frequency of experimentally manipulated magnitude and parity changes, in a passive viewing setting. Similar FPVS paradigms relying on passive viewing have successfully been used to study high-level discrimination of faces $^{78}$, tools ${ }^{79}$, as well as reading (word recognition ${ }^{80}$, and letter strings discrimination ${ }^{69,81}$ ), and non-symbolic quantities ${ }^{82}$, without engaging participants in any kind of explicit processing of the presented stimuli.

We combined the FPVS paradigm with a standard/deviant procedure (oddball method $\left.{ }^{83}\right)$. More precisely, during one-minute sequences, we displayed digits at the very fast rate of $10 \mathrm{~Hz}$ (ten stimuli per second) following a sinusoidal contrast modulation. Within the stream of standard digits, a periodic deviant was introduced every eight trials such that digits from the deviant category appeared at $1.25 \mathrm{~Hz}$ (see Fig. 1). The digits used as standards or deviants varied according to the condition: In the magnitude condition, digits smaller than five were 
a
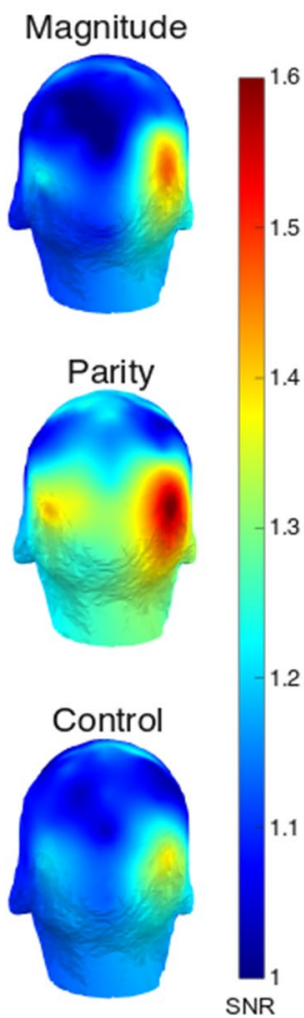

b
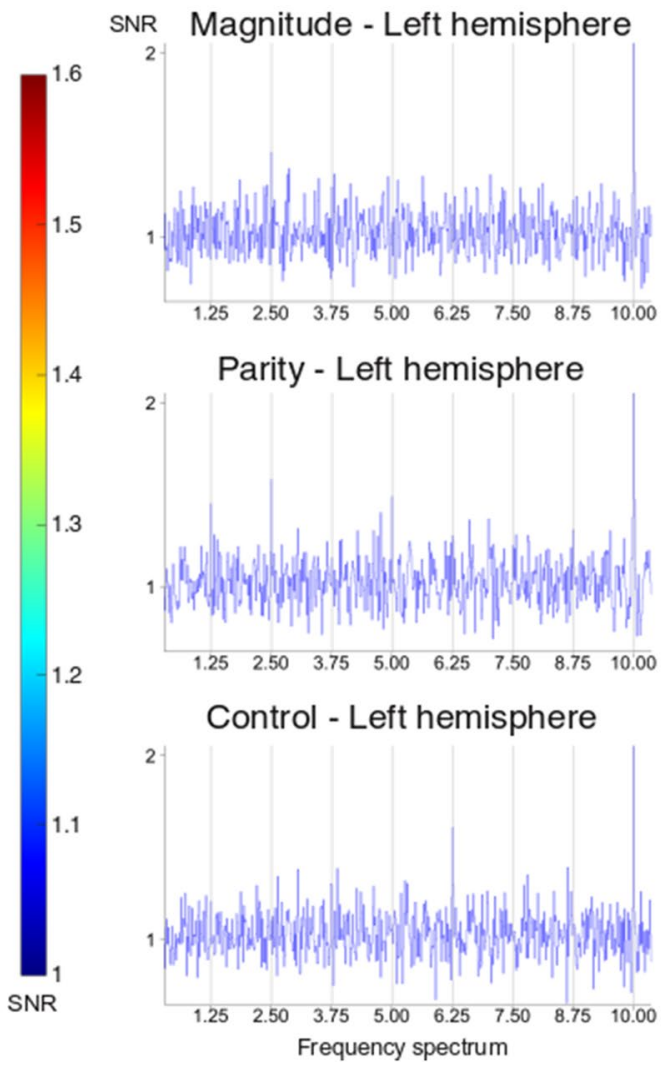
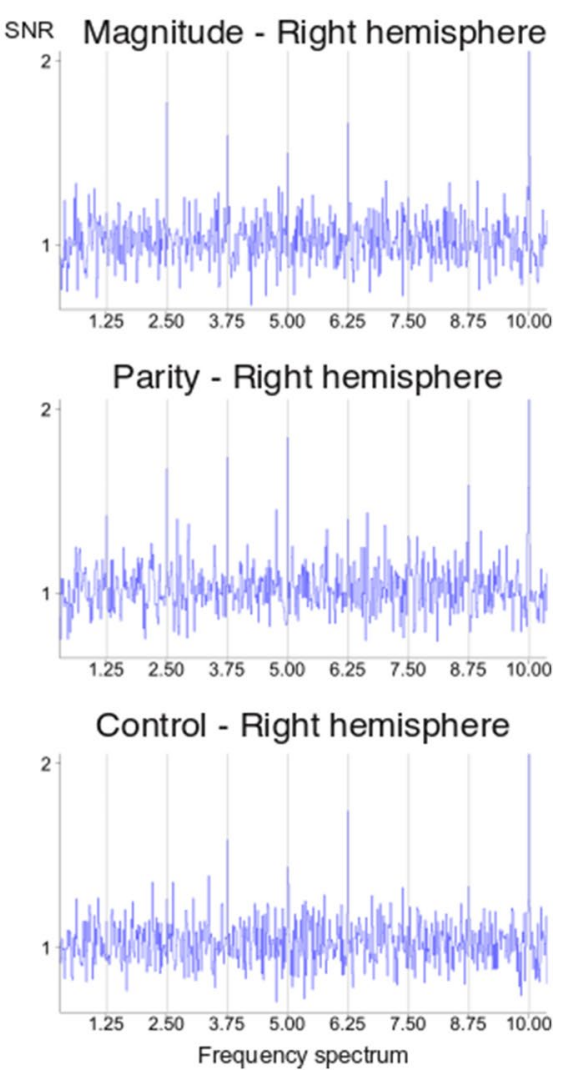

Figure 2. (a) Topographical maps of the periodic brain responses expressed as the Signal-to-Noise Ratio (SNR) of the average of the amplitude at the oddball frequency (1.25 Hz and its harmonics up to the 7th). (b) Amplitudes spectra expressed as SNR for the three conditions (magnitude, parity, and control) within the left electrodes of interest (A9, A10, A11) and within the right electrodes of interest (B6, B7, B8).

presented as standard and digits larger than five as deviants, or the other way around. In the parity condition, odd digits were presented as standards and even digits as deviants, or the other way around. Finally, in a control condition, half of the digits were arbitrary categorized as standards and the other half as deviants (see Fig. 1).

We postulate that we should record an electrophysiological response synchronized at the deviation frequency (i.e., $1.25 \mathrm{~Hz}$ ) if the brain is sensitive to the changes from one category to another. In other words, if magnitude and/or parity are processed in an autonomous unintentional automatic way, then the category changes should systematically elicit a specific brain response at the frequency of the changes for the respective condition(s), while no such response should arise during the control condition as the latter's digit categories were arbitrary and not related to any semantic content. Doing so, we can objectively measure whether a given semantic number information is automatically and unintentionally processed by the brain although no numerical task was requested from the participant.

\section{Results}

Figure 2a illustrates the topographical maps of the averaged oddball amplitudes for every condition, expressed as the Signal-to-Noise Ratio (SNR) of the frequency bin of interest and its harmonics up to the seventh (i.e., $1.25,2.50,3.75,5.00,6.25,7.50$, and $8.75 \mathrm{~Hz}$ ). These maps highlight that the strongest recorded responses in each condition were located over a bilateral region encompassing occipitoparietal areas. It is worth noticing that there was a substantial overlap of the location of the brain responses between the experimental conditions, which allows direct comparison of amplitudes recorded within these regions.

Figure $2 \mathrm{~b}$ represents the EEG spectra recorded on the electrodes from the left (A9, A10, A11) and right (B6, $\mathrm{B} 7, \mathrm{~B} 8$ ) occipitoparietal regions of interest corresponding to the topographical maps. The spectra, expressed as SNRs, are averaged across participants for every condition. In each condition, there were clear peaks at $10 \mathrm{~Hz}$, with Z-scores systematically reaching values larger than five. These findings support that both occipitoparietal regions specifically synchronised to the $10 \mathrm{~Hz}$ base rate during the recording sessions. Note, however, that neural synchronisation to the base rate merely reflects brain response to the periodic stimulus onsets at $10 \mathrm{~Hz}$.

More critically for the purpose of the current study, we also observed in Fig. 2b large peaks in the frequency bins that are harmonics of the periodic category fluctuation (up to the seventh harmonic). In the Magnitude condition, there were fewer clear peaks across these frequency bins within the left electrodes, but peaks were 


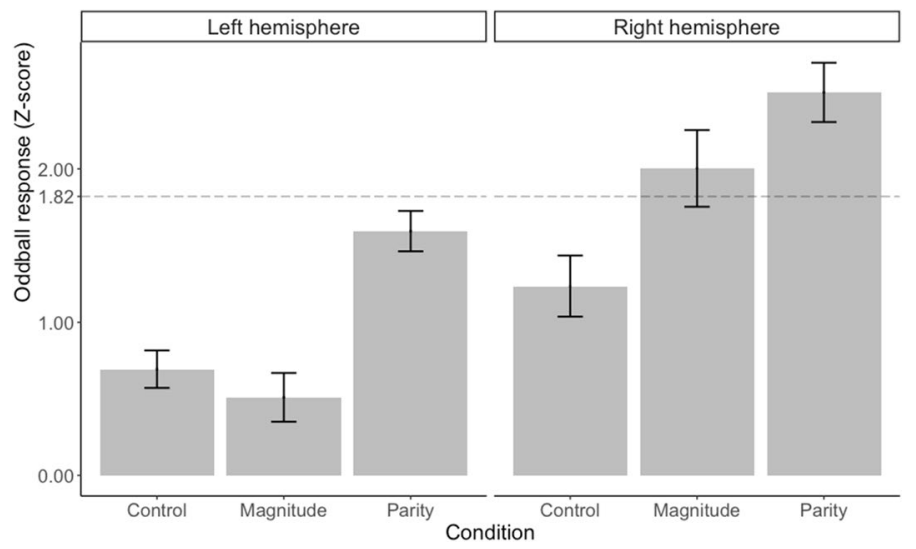

Figure 3. Mean amplitude, in Z-score, across the oddball frequency and its harmonics up to the seventh, as a function of the condition and as a function of the electrode lateralisation (left hemisphere: A9, A10, A11; right hemisphere: B6, B7, B8). Vertical bar depicts standard deviations. The horizontal dashed line represents the onesided $95 \%$ threshold of significance $(Z>1.82, p<.05$, one-tailed $)$.

clearly depicted in the right hemisphere. For the Parity condition, we observed some clear peaks within the left region of interest, and many definite peaks within the right region of interest. Finally, more unexpectedly, we also found some responding peaks in the control condition, mostly located within the right occipitoparietal regions.

In order to assess whether these peaks were statistically significant, we computed the average signal amplitude of the frequency of interest and its harmonics up to the seventh, expressed as a Z-score, as a function of the condition (see Fig. 3). Within the three left electrodes of interest, mean oddball amplitude during the Magnitude condition was 0.51 , Standard Deviation $(S D)=1.52$, mean amplitude in the Parity condition was $1.59, S D=1.26$, and mean amplitude for the Control condition was $0.69, S D=1.17$. Within the right electrodes, mean amplitude during the Magnitude condition was 2.01, $S D=2.40$, during the Parity condition it reached $2.50, S D=1.85$, and it was $1.23, S D=1.90$, for the Control condition. Visual inspection of Fig. 3 reveals that the right occipitoparietal regions of interest were significantly sensitive to the periodic category changes during both experimental conditions, with z-scored amplitudes exceeding the critical threshold of 1.82 corresponding to the 95th percentile in our skewed distribution.

In order to compare brain responses between conditions, we performed a repeated-measures ANOVA with the region of interest (two levels, either left or right) and the condition (three levels) as fixed factors. There was a significant main effect of the region factor, $F(1,44)=5.468, p=0.024$, partial $\eta^{2}=0.111$, with stronger amplitudes in the right $\mathrm{ROI}($ mean $=1.91, \mathrm{SD}=2.10)$ than in the left $\mathrm{ROI}($ mean $=0.93, \mathrm{SD}=1.39$ ). There was also a significant main effect of the condition factor, $F(2,88)=14.409, p<0.001$, partial $\eta^{2}=0.179$, with stronger responses in the Parity condition $($ mean $=2.04, \mathrm{SD}=1.63)$ than in the Magnitude condition $(\mathrm{mean}=1.25, \mathrm{SD}=2.12)$ and the Control condition (mean $=0.96, \mathrm{SD}=1.58$ ). The interaction did not reach the significance level, $F(2,88)=1.761$, $p=0.178$, partial $\eta^{2}=0.038$. We still decided to conduct post-hoc comparison analyses to better characterize the different response patterns in each ROI illustrated in Fig. 3. We thus conducted pairwise Holm-Bonferroni corrected Wilcoxon tests to directly compare the condition levels between each other. These tests first revealed that responses were stronger in the right ROI than in the left ROI for both experimental conditions $(W=37, p=0.014$; $W=16, p<0.001)$, but not for the control condition $(W=99, p=0.741)$. Secondly, they revealed that, for the left electrodes of interest, Parity yielded significantly greater responses than both Control, $W=56, p=0.050$, and Magnitude conditions, $W=43, p=0.014$. On the other hand, Magnitude and Control did not differ from each other, $W=168, p=0.754$. For the right electrodes of interest, Parity and Magnitude were significantly greater than the control condition, respectively $W=35, p=0.012$, and $W=65, p=0.025$, but the experimental conditions did not differ from each other, $W=91, p=0.640$.

\section{Discussion}

Magnitude and parity are very salient features of number symbols ${ }^{5,30}$. While there are several studies demonstrating the existence of automatic number symbols processing in educated adults, only a few studies could unequivocally demonstrate the existence of autonomous and unintentional number magnitude processing. Therefore, it is still necessary to provide further evidence by triggering magnitude as little as possible in order to disentangle autonomous from intentional automatic processing ${ }^{21}$. Moreover, data concerning an automatic processing of parity are currently lacking.

The current FPVS paradigm allows investigating the spontaneous and autonomous nature of specific cognitive processes during passive viewing of rapid sequence ${ }^{84}$. This technique has not yet been used to investigate number symbol processing, though it is very relevant for our objective since it is an objective measure of the brain response at a frequency defined a priori by the experimenter, which allows direct and straightforward analysis of the neural synchronisation. Moreover, the recorded responses quantify the processing of digits' magnitude and parity without an active task and thus, without the implication of decision-making processes ${ }^{85}$. Taken together, the current design avoided any conscious triggering of magnitude or parity during the recording session and 
thus probe the autonomous nature of these numerical processes. This is supported by the fact that no participant reported detecting any pattern or any periodicity in the visual stimulations.

We observed a significant neural synchronisation to changes in numerical magnitude and parity within right occipitoparietal areas, whereas no such synchronisation was found during the control condition. It is noteworthy that visual inspection of SNRs suggests that some brain responses peaked at some harmonics of interest in the control condition, where category was based on temporarily constructed rules, but these peaks did not reach the statistical significance. In contrast, the strong responses within the right occipitoparietal electrodes indicate that participants implicitly processed the category to which numerical stimuli belong and reacted to changes with respect to the category. Additionally, parity yielded significantly larger brain responses than the other conditions in the left hemisphere.

We thus measured substantial implicit responses to the two manipulated semantic features, but not to shortterm arbitrary associations. This means that we can exclude that the brain synchronizations recorded in both experimental tasks were only due to the rare nature of the deviant stimulus. These evidences support the view that both numerical magnitude and parity were automatically accessed and processed during passive viewing sessions. In other words, both semantic features are spontaneously and unintentionally extracted from Arabic digits, which corroborates previous observations that several deliberate tasks on Arabic digits (such as naming) are necessarily semantically mediated ${ }^{14}$. It is noteworthy that the automaticity of parity that we observed here does not support the hypothesis arguing that parity is computed by dividing the number by two ${ }^{59}$, but our findings are rather in line with the idea of a direct retrieval from semantic memory ${ }^{60,61}$.

The topography of the frequency-tagged responses corresponds with seminal models of numerical cognition, such as the triple-code model ${ }^{70,86}$, since we found significant responses over right occipitoparietal electrodes specifically related to both magnitude and parity changes. This is in line with the hypothesis that the semantic of number representations are housed in parietal regions ${ }^{37}$. The occipitoparietal responses that we obtained during Magnitude and Parity conditions are typical in ERP studies investigating the neural distance effect with Arabic numbers ${ }^{68,87,88}$. These studies highlighted a neural distance effect illustrated by a modulation of the positivity of $\mathrm{P} 2 \mathrm{p}$ recorded over occipitoparietal electrodes as a function of the numerical distance during numerical comparison. These studies, except ${ }^{88}$, also found a dominance of the right hemisphere for numerical magnitude comparison. In the absence of an active task, we similarly found significant right-sided dominance for numerical symbols' processing. We did not, however, observe any significant differences between right hemisphere electrodes responding to parity and magnitude, which is partly in line with a PET study indicating that parity and magnitude information are processed in neighbouring area inside the IPS ${ }^{89}$.

Finally, it is noteworthy that each digit presentation only lasted $100 \mathrm{~ms}$ in the current experimental design (since our base rate was $10 \mathrm{~Hz}$, see Fig. 1). Previous ERP studies showed that number processing actually follows a time course that involves distinct stages of neural processes ${ }^{90}$. Critically, numerical magnitude was already reported to be early extracted (within $200 \mathrm{~ms}$ ) from digit symbols ${ }^{90}$. Due to the fast display period in the current FPVS paradigm, fewer than $100 \mathrm{~ms}$, we can assume that our method mostly captured early cognitive processes. Because we observed significant brain synchronization to magnitude and to parity, our results consequently support that not only magnitude, but also parity information, can be extracted and processed very early in the brain. The time course of the autonomous processing of numerical features could be investigated in future studies by, for instance, varying the base rate of similar FPVS paradigms. More generally, the current paradigm provides strong evidence of unintentional and autonomous processing of semantic information from digits, and it could be easily implemented to follow how symbols' semantic processing becomes automatic across typical and atypical development.

In summary, we observed significant brain responses tagged at the frequency of magnitude and parity periodic changes over occipitoparietal electrodes during passive viewing of a rapid stream of Arabic digits. We did not observe significant brain responses to arbitrarily constructed number categories in our control condition. Critically, in our FPVS design, the digit display time was under $100 \mathrm{~ms}$, which is too short for deliberate semantic processing ${ }^{87,90}$, and participants did not report conscious detection of the periodic changes. We consequently argue that such frequency-tagged brain responses were automatic and not goal-driven. Our findings are thus is line with previous reports that numerical magnitude is spontaneously processed, and we also provide evidence that parity is a semantic feature that is unintentionally activated by Arabic digits in numerate adults. The current study thus provides evidence that culturally learned number symbols become automatically processed by the human mind.

\section{Method}

Participants. In order to determine the sample size, we considered the study from Park (2017) where he reported an effect size of 0.18 for neural synchronizations to non-symbolic quantities within posterior regions. With an alpha of 0.05 and a power of 0.95 , a sample size of $n=17$ should be sufficient to detect numerical synchronization.

Twenty-nine undergraduate students from the University of Luxembourg participated in the study. Any history of neurological or neuropsychological disease or any uncorrected visual impairment constituted exclusion criteria. To ensure participants had no major mathematical difficulties, participants' arithmetic fluency was evaluated with the Tempo-Test Rekenen ${ }^{91}$, which is a timed pen-and-paper test (five minutes) consisting in arithmetic problems of increasing difficulty. All participants reached the inclusion criterion, which was 100 correct items out of 200, and were included into the present study. Six participants were excluded from the final sample due to substantial noise in their EEG signal (mostly due to transpiration). The final sample thus consisted in 23 adults, with a mean age of 24 years $(S D=3.5)$. This sample size is larger than the minimal size that we previously computed. Participants received 30 euros for their participation. 
Experimental setup. We used MATLAB (The MathWorks) with the Psychophysics Toolbox extensions ${ }^{92-94}$ to display the stimuli and record behavioural data. The EEG recording took place in a shielded Faraday cage $(288 \mathrm{~cm} \times 229 \mathrm{~cm} \times 222 \mathrm{~cm})$. Participants were seated at $1 \mathrm{~m}$ from the screen, with their eyes perpendicular to the centre of the screen (24' LED monitor, $100 \mathrm{~Hz}$ refresh rate, $1 \mathrm{~ms}$ response time). Screen resolution was $1024 \times 768 \mathrm{px}$, with a light grey background colour. The order of the conditions during the EEG recording session was counterbalanced across participants.

Material and procedure. Although FPVS paradigm theoretically only involves passive viewing of the stimuli, we introduced a basic orthogonal active task during the recording sessions in order to ascertain that participants were looking at the computer screen. Participants were thus instructed to fixate the centre of the screen where a small blue diamond (12px size) was displayed, and they were asked to press a button with their right forefinger when they detected that the diamond changed its colour from blue to red. This colour change was not periodic and could randomly occur six to eight times in a given sequence. Participants were also informed that black digits ranging from 1 to 9 - excluding 5 - would quickly appear on the screen. They were explicitly instructed not to actively look at the digits but to keep their gaze on the central diamond. On average, participants took $640 \mathrm{~ms}(S D=121 \mathrm{~ms})$ to respond to the colour change that affected the fixation diamond. Misses were very rare, occurring only in $1.5 \%$ of the trials. Such high detection rate indicates that participants followed the instruction and kept their gaze on the centre of the screen during EEG acquisition.

Digits were sequentially presented at the fast base frequency of $10 \mathrm{~Hz}$ (i.e., ten stimuli per second) following a sinusoidal contrast modulation from 0 to $100 \%{ }^{78,82,95}$ (see Fig. 1). There was thus for each stimulus a $50 \mathrm{~ms}$ period of gradual fade-in and $50 \mathrm{~ms}$ of gradual fade-out. The successive presentation of digits consisted in sequences that lasted $64 \mathrm{~s}$, including $60 \mathrm{~s}$ of stimulation and $2 \mathrm{~s}$ of fade-in and fade-out, which we did not analyse.

We used the FPVS variation of the oddball design ${ }^{83}$ in which we introduced a periodic fluctuation within the standard sequence: the stimulus category changed every eight items (i.e., at $1.25 \mathrm{~Hz}$ ). Our design included three different experimental conditions, each consisting in a different category change. In the magnitude condition, periodical variations were based on the magnitude of the Arabic digit (i.e., smaller than 5 or larger than 5). For half of the sequences, the standard stimuli displayed at the base rate were randomly drawn among the smallest numbers $(1,2,3,4)$ whereas the deviant item was randomly drawn among the largest numbers $(6,7,8,9)$. For the other half, it was the opposite, the greatest numbers were standards and the smallest ones were deviant. In the parity condition, periodical variation was based on the parity of the Arabic digit (i.e., odd vs. even). For half of the sequences, the odd category $(1,3,7,9)$ was standard and the even category $(2,4,6,8)$ was deviant, and vice-versa for the other half. Finally, we introduced a control condition that was based on two arbitrary categories with equal amounts of odd, even, small and large numbers $(1,4,6,9$ vs. 2, 3, 7, 8). As the control categories were arbitrary, and in order to reduce the recording time, we only presented the first set as the standard category and the second set as the deviant category. Sequences for every condition were repeated four times. The magnitude and parity conditions each yielded eight sequences, for a total of twenty sequences. If visual inspection of the signal during acquisition led to detecting obvious offsets of the electrodes (e.g., movement), the experimenter excluded and reran the noisy sequence. This procedure occurred in less than $1 \%$ of the sequences.

Following previous recommendations ${ }^{84}$, and to decrease habituation to the visual properties of the stimuli ${ }^{9}$, we deliberatively introduced stochastic physical variations within the stimuli stream. The stimulus font randomly varied among four possibilities (Arial, Times New Roman, Cambria, and Calibri), the position of the stimulus randomly varied on both the vertical and horizontal axes (with a variation of maximum $10 \%$ from the centre of the screen), and the font size varied (from 122 to 148, with an average value of 135). These random visual variations occurred at each onset and were thus not congruent with our frequencies of interest (i.e., $1.25 \mathrm{~Hz}$ and its harmonics).

EEG acquisition. We used a 128-channel BioSemi ActiveTwo system (BioSemi B. V., Amsterdam, The Netherlands) tuned at $1024 \mathrm{~Hz}$ to acquire EEG data, as $\mathrm{in}^{82}$. We did not filter the signal on-line, except for using default low-pass anti-aliasing filter settings. We positioned the electrodes on the cap according to the standard 10-20 system locations (for exact position coordinates and for more information about default anti-aliasing filtering settings, see http://www.biosemi.com). We used two supplementary electrodes, the Common Mode Sense (CMS) active electrode and the Driven Right Leg (DRL) passive electrode, as reference and ground electrodes, respectively. We held electrodes offsets (referenced to the CMS) below $40 \mathrm{mV}$. We also monitored eye movements with four flat-type electrodes; two were positioned lateral to the external canthi, the other two placed above and below participant's right eye; but we did not further analyse these electrodes.

EEG analysis. Analyses were conducted with the help of Letswave 6 (http://nocions.webnode.com/letsw ave). Before starting the analyses, we down-sampled our data file resolution from 1024 to $512 \mathrm{~Hz}$ for faster computer processing. We used a 4-order band-pass Butterworth filter $(0.1$ to $100 \mathrm{~Hz})$ and we then re-referenced the data to the common average. We did not interpolate any electrode. We did not correct the EEG signal for the presence of ocular artefacts because the FPVS approach is robust to these artefacts. Indeed, the narrow frequency bins of interest make the signal in the frequency domain largely immune to eyeblinks ${ }^{85}$. The fade-in and the fadeout periods were excluded from the analyses leading to the segmentation of an EEG signal of $60 \mathrm{~s}$ (corresponding to the display of 600 stimuli). We averaged the signal from all repetitions of each condition for each participant. Note that we did not expect that brain responses to deviant odd digits within a sequence of standard even digits would be different than brain responses to deviant even digits within a sequence of standard odd digits in the Parity condition (and similarly for small and large numbers in the Magnitude condition). We thus aggregated all repetitions for these conditions. Finally, we performed a Fast Fourier Transformation (FFT) on the averaged 
signals, and we extracted amplitude spectra for the 128 channels with a frequency resolution (the size of the frequency bins) of $0.016 \mathrm{~Hz}$.

Based on the frequency spectra, we computed two measures to determine whether and how the brain specifically responded to the deviant frequency during the three manipulated conditions. Following the standard procedures deployed in recent FPVS paradigms ${ }^{80,82,84,85,95-99}$, we computed (a) the Signal-to-Noise Ratios (SNRs) to illustrate the frequency spectra in all conditions, and (b) Z-scores to provide straightforward statistical information about the significance of the observed brain responses.

We computed the SNRs by dividing each frequency bin by the mean amplitude of their respective twenty surrounding bins (ten on each side, excluding the immediately adjacent bins, and the two most extreme values $^{78,82,96,100,101}$ ). In particular, we considered the signal recorded from the twenty surrounding bins as representative of the noise level. If a given frequency bin yields larger responses than the noise level, the SNR should be larger than 1. Conversely, if a given frequency bin does not yield larger responses than the noise level, the SNR should tend to be 1 . We used SNRs to depict the frequency spectra and illustrate the topographies of our results (see Fig. 2). Given the topography of the SNR data (see Fig. 2a), we merged some electrodes into two regions of interest: we subsequently computed left (A9, A10, A11) and right (B6, B7, B8) occipitoparietal areas of interest.

We also computed a Z-score to assess the statistical significance of the brain responses to the category change. To do so, for each condition and for each participant, we cropped the FFT spectra around the frequency of interest $(1.25 \mathrm{~Hz})$ and its subsequent harmonics up to the seventh (i.e., 1.25, 2.5, 3.75, 5, 6.25, 7.5, and $8.75 \mathrm{~Hz}$ ) surrounded by their twenty respective neighbouring bins (ten on each side ${ }^{82}$ ). We summed all cropped spectra and then applied a Z-transformation to the amplitudes. We finally extracted from this Z-transformation the value of the merged critical bin of interest. This value represents the brain response specific to the experimental manipulation at $1.25 \mathrm{~Hz}$ (and its harmonics). This score can then be interpreted as the strength of the neural detection of the change. As a Z-score, a value larger than the value corresponding to the 95th percentile indicates a significant response to the change $(p<0.05$, one-tailed, testing signal level $>$ noise level). As noted by an anonymous reviewer, the amplitudes distribution was skewed here because the amplitudes were moduli of Fourier components, which cannot be negative. Simulations indeed confirmed that the critical value corresponding to the 95th percentile in such a distribution was higher, approximating 1.82. We thus used 1.82 as our critical threshold to assess statistical significance. Lastly, we used Z-scores to directly compare the strengths of the electrophysiological responses across conditions.

\section{Ethical considerations}

All procedures performed in this study were in accordance with the ethical standards of the APA, and with the 1964 Helsinki Declaration and its later amendments or com- parable ethical standards. The Ethic Review Panel from the University of Luxembourg approved the methodology and the implementation of the experiment before the start of data collection. Informed consent was obtained from all individual participants included in the study.

Received: 27 December 2019; Accepted: 20 November 2020

Published online: 17 December 2020

\section{References}

1. Gilmore, C., Göbel, S. M. \& Inglis, M. An Introduction to Mathematical Cognition. Matthew Inglis. Description (Routledge, New York, NY, 2018). https://doi.org/10.4324/9781315684758.

2. Benoit, L., Lehalle, H. \& Jouen, F. Do young children acquire number words through subitizing or counting?. Cogn. Dev. 19, 291-307. https://doi.org/10.1016/j.cogdev.2004.03.005 (2004).

3. Benoit, L., Lehalle, H., Molina, M., Tijus, C. \& Jouen, F. Young children's mapping between arrays, number words, and digits. Cognition 129(1), 95-101. https://doi.org/10.1016/j.cognition.2013.06.005 (2013).

4. Berch, D. B., Foley, E. J., Hill, R. J., Ryan, P. M. \& McDonough Ryan, P. Extracting parity and magnitude from Arabic numerals: developmental changes in number processing and mental representation. J. Exp. Child Psychol. 74(4), 286-308. https://doi. org/10.1006/jecp.1999.2518 (1999).

5. Miller, K. F. What A Number is: Mathematical Foundations and Developing Number Concepts. In Advances in psychology Vol. 91 (ed. Campbell, J. I. D.) 3-38 (Elsevier, Amsterdam, 1992). https://doi.org/10.1016/S0166-4115(08)60883-3.

6. Miura, I. T. et al. First graders' cognitive representation of number and understanding of place value: cross-national comparisons: France, Japan, Korea, Sweden, and the United States. J. Educ. Psychol. 85(1), 24-30. https://doi.org/10.1037/0022-0663.85.1.24 (1993).

7. Moeller, K., Pixner, S., Zuber, J., Kaufmann, L. \& Nuerk, H.-C. Early place-value understanding as a precursor for later arithmetic performance-a longitudinal study on numerical development. Res. Dev. Disabil. 32(5), 1837-1851. https://doi.org/10.1016/j. ridd.2011.03.012 (2011).

8. Cipora, K., Soltanlou, M., Smaczny, S., Melanie Goebel, S. \& Nuerk, H.-C. Automatic place-value activation in magnitudeirrelevant parity judgement. Psychol. Res. https://doi.org/10.1007/s00426-019-01268-1 (2018).

9. Cohen, D. J. Integers do not automatically activate their quantity representation. Psychon. Bull. Rev. 16(2), 332-336. https://doi. org/10.3758/PBR.16.2.332 (2009).

10. Cohen Kadosh, R., Bien, N. \& Sack, A. T. Automatic and intentional number processing both rely on intact right parietal cortex: a combined FMRI and neuronavigated TMS study6, 2. Front. Hum. Neurosci. https://doi.org/10.3389/fnhum.2012.00002 (2012).

11. Dehaene, S. \& Akhavein, R. Attention, automaticity, and levels of representation in number processing. J. Exp. Psychol. Learn. Mem. Cogn. 21(2), 314-326. https://doi.org/10.1037/0278-7393.21.2.314 (1995).

12. Dormal, V. \& Pesenti, M. Processing numerosity, length and duration in a three-dimensional Stroop-like task: towards a gradient of processing automaticity?. Psychol. Res. https://doi.org/10.1007/s00426-012-0414-3 (2012).

13. Duncan, E. M. \& McFarland, C. E. Isolating the effects of symbolic distance, and semantic congruity in comparative judgments: an additive-factors analysis. Memory Cogn. 8(6), 612-622. https://doi.org/10.3758/BF03213781 (1980).

14. Fias, W., Reynvoet, B. \& Brysbaert, M. Are Arabic numerals processed as pictures in a Stroop interference task?. Psychol. Res. 65(4), 242249. https://doi.org/10.1007/s004260100064 (2001).

15. Girelli, L., Lucangeli, D. \& Butterworth, B. The development of automaticity in accessing number magnitude. J. Exp. Child Psychol. 76(2), 104-122. https://doi.org/10.1006/jecp.2000.2564 (2000). 
16. Henik, A. \& Tzelgov, J. Is three greater than five: the relation between physical and semantic size in comparison tasks. Memory Cogn. 10(4), 389-395. https://doi.org/10.3758/BF03202431 (1982).

17. Kallai, A. Y. \& Tzelgov, J. The place-value of a digit in multi-digit numbers is processed automatically. J. Exp. Psychol. Learn. Mem. Cogn. 38(5), 1221-1233. https://doi.org/10.1037/a0027635 (2012).

18. Pansky, A. \& Algom, D. Comparative judgment of numerosity and numerical magnitude: attention preempts automaticity. J. Exp. Psychol. Learn. Mem. Cogn. 28(2), 259-274. https://doi.org/10.1037/0278-7393.28.2.259 (2002).

19. Rubinsten, O. \& Henik, A. Automatic activation of internal magnitudes: a study of developmental Dyscalculia. Neuropsychology 19(5), 641-648. https://doi.org/10.1037/0894-4105.19.5.641 (2005).

20. Suzcs, D., Soltész, F., Jármi, É. \& Csépe, V. The speed of magnitude processing and executive functions in controlled and automatic number comparison in children: An electro-encephalography study. Behav. Brain Funct. 3(1), 23. https://doi.org/10.1186/17449081-3-23 (2007).

21. Tzelgov, J. \& Ganor-Stern, D. Automaticity in processing ordinal information. In Handbook of Mathematical Cognition (ed. Campbell, J. I. D.) 55-66 (Psychology Press, New York, 2005).

22. Tzelgov, J., Meyer, J. \& Henik, A. Automatic and intentional processing of numerical information. J. Exp. Psychol. Learn. Mem. Cogn. 18(1), 166-179. https://doi.org/10.1037/0278-7393.18.1.166 (1992).

23. Booth, J. L. \& Siegler, R. S. Numerical magnitude representations influence arithmetic learning. Child Dev. 79(4), 1016-1031 (2008).

24. Bugden, S., Price, G. R., McLean, D. A. \& Ansari, D. The role of the left intraparietal sulcus in the relationship between symbolic number processing and children's arithmetic competence. Dev. Cogn. Neurosci. 2(4), 448-457. https://doi.org/10.1016/j. den.2012.04.001 (2012).

25. Butterworth, B., Zorzi, M., Girelli, L. \& Jonckheere, A. R. Storage and retrieval of addition facts: the role of number comparison. Q. J. Exp. Psychol. Sect. A: Hum. Exp. Psychol. 54(4), 1005-1029. https://doi.org/10.1080/713756007 (2001).

26. Cohen Kadosh, R. et al. Virtual Dyscalculia induced by parietal-lobe TMS impairs automatic magnitude processing. Curr. Biol. 17(8), 689-693. https://doi.org/10.1016/j.cub.2007.02.056 (2007).

27. De Smedt, B., Noël, M. P., Gilmore, C. \& Ansari, D. How do symbolic and non-symbolic numerical magnitude processing skills relate to individual differences in children's mathematical skills? A review of evidence from brain and behavior. Trends Neurosci. Educ. https://doi.org/10.1016/j.tine.2013.06.001 (2013).

28. Fazio, L. K., Bailey, D. H., Thompson, C. A. \& Siegler, R. S. Relations of different types of numerical magnitude representations to each other and to mathematics achievement. J. Exp. Child Psychol. https://doi.org/10.1016/j.jecp.2014.01.013 (2014).

29. Leibovich, T., Katzin, N., Harel, M. \& Henik, A. From 'sense of number' to 'sense of magnitude'-the role of continuous magnitudes in numerical cognition. Behav. Brain Sci. https://doi.org/10.1017/S0140525X16000960 (2016).

30. Miller, K. \& Gelman, R. The child's representation of number: a multidimensional scaling analysis. Child Dev. 54(6), 1470-1479. https://doi.org/10.2307/1129809 (1983).

31. Posner, M. I. Chronometric Explorations of Mind Vol. 286 (Oxford University Press, Oxford, 1978) https://doi.org/10.1038/ncb32 41.

32. Tzelgov, J. Automatic but conscious: that is how we act most of the time. In The Automaticity of Everyday Life: Advances in Social Cognition Vol. 10 (ed. Wyer, R. S., Jr.) (Psychology Press, New York, 1997).

33. Tzelgov, J., Yehene, V. \& NavehBenjamin, M. From Memory to Automaticity and "Vice Versa": On the Relations Between Memory and Automaticity. In Idealization VIII: Modelling in Psychology (eds Brzeziński, J. et al.) 239 (Brill, Leiden, 1997).

34. Evans, J. S. B. On the resolution of conflict in dual process theories of reasoning. Think. Reason. 13, 321-339. https://doi. org/10.1080/13546780601008825 (2007).

35. Evans, J. S. B. Dual-processing accounts of reasoning, judgment, and social cognition. Annu. Rev. Psychol. 59, 255-278. https:// doi.org/10.1146/annurev.psych.59.103006.093629 (2008).

36. Evans, J. S. B. \& Stanovich, K. E. Dual-process theories of higher cognition: advancing the debate. Perspect. Psychol. Sci. 8, 223-241. https://doi.org/10.1177/1745691612460685 (2013).

37. Dehaene, S., Piazza, M., Pinel, P. \& Cohen, L. Three parietal circuits for number processing. Cogn. Neuropsychol. $20(36), 487506$. https://doi.org/10.1080/02643290244000239 (2003).

38. Tzelgov, J., Yehene, V., Kotler, L. \& Alon, A. Automatic comparisons of artificial digits never compared: learning linear ordering relations. J. Exp. Psychol.: Learn. Memory Cogn. https://doi.org/10.1037/0278-7393.26.1.103 (2000).

39. Restle, F. Speed of adding and comparing numbers. J. Exp. Psychol. 83(2 PART 1), 274-278. https://doi.org/10.1037/h0028573 (1970).

40. Gevers, W., Verguts, T., Reynvoet, B., Caessens, B. \& Fias, W. Numbers and space: a computational model of the SNARC effect. J. Exp. Psychol. Hum. Percept. Perform. 32(1), 32-44. https://doi.org/10.1037/0096-1523.32.1.32 (2006).

41. Santens, S. \& Gevers, W. The SNARC effect does not imply a mental number line. Cognition 108(1), 263-270. https://doi. org/10.1016/j.cognition.2008.01.002 (2008).

42. Proctor, R. W. \& Cho, Y. S. Polarity correspondence: a general principle for performance of speeded binary classification tasks. Psychol. Bull. https://doi.org/10.1037/0033-2909.132.3.416 (2006).

43. Abrahamse, E., van Dijck, J.-P. \& Fias, W. How does working memory enable number-induced spatial biases?. Front. Psychol. 7(JUNE), 977. https://doi.org/10.3389/fpsyg.2016.00977 (2016).

44. Fias, W. \& van Dijck, J.-P. The temporary nature of number-space interactions. Can. J. Exp. Psychol./Revue Canadienne de Psychologie Expérimentale 70(1), 33-40. https://doi.org/10.1037/cep0000071 (2016).

45. van Dijck, J.-P. \& Fias, W. A working memory account for spatial-numerical associations. Cognition 119(1), 114-119. https:// doi.org/10.1016/j.cognition.2010.12.013 (2011).

46. van Dijck, J.-P., Gevers, W., Lafosse, C. \& Fias, W. The Heterogeneous nature of number-space interactions. Front. Hum. Neurosci. 5, 182. https://doi.org/10.3389/fnhum.2011.00182 (2012).

47. van Dijck, J. P., Abrahamse, E. L., Acar, F., Ketels, B. \& Fias, W. A working memory account of the interaction between numbers and spatial attention. Q. J. Exp. Psychol. 67(8), 1500-1513. https://doi.org/10.1080/17470218.2014.903984 (2014).

48. van Dijck, J. P., Gevers, W. \& Fias, W. Numbers are associated with different types of spatial information depending on the task. Cognition 113(2), 248-253. https://doi.org/10.1016/j.cognition.2009.08.005 (2009).

49. Lammertyn, J., Wim, F. \& Lauwereyns, J. Semantic influences on feature-based attention due to overlap of neural circuits. Cortex 38(5), 878-882. https://doi.org/10.1016/S0010-9452(08)70061-3 (2002).

50. Roettger, T. B. \& Domahs, F. Grammatical number elicits SNARC and MARC effects as a function of task demands. Q. J. Exp. Psychol. 68(6), 1231-1248. https://doi.org/10.1080/17470218.2014.979843 (2015).

51. Fischer, M. H. Number processing induces spatial performance biases. Neurology 57(5), 822-826. https://doi.org/10.1212/ WNL.57.5.822 (2001).

52. Keus, I. M. \& Schwarz, W. Searching for the functional locus of the SNARC effect: evidence for a response-related origin. Memory Cogn. 33(4), 681-695. https://doi.org/10.3758/BF03195335 (2005).

53. Hoffmann, D., Hornung, C., Martin, R. \& Schiltz, C. Developing number-space associations: SNARC effects using a color discrimination task in 5-year-olds. J. Exp. Child Psychol. 116(4), 775-791. https://doi.org/10.1016/j.jecp.2013.07.013 (2013).

54. Stroop, J. R. Studies of interference in serial verbal reactions. J. Exp. Psychol. 18(6), 643-662. https://doi.org/10.1037/h0054651 (1935). 
55. Besner, D. \& Coltheart, M. Ideographic and alphabetic processing in skilled reading of English. Neuropsychologia 17(5), 467-472. https://doi.org/10.1016/0028-3932(79)90053-8 (1979).

56. Vaid, J. \& Corina, D. Visual field asymmetries in numerical size comparisons of digits, words, and signs. Brain Lang. 36(1), 117-126. https://doi.org/10.1016/0093-934X(89)90055-2 (1989).

57. Moyer, R. S. \& Landauer, T. K. Time required for judgements of numerical inequality. Nature 215(5109), 1519-1520. https:// doi.org/10.1038/2151519a0 (1967)

58. Yang, T. et al. Development of spatial representation of numbers: a study of the SNARC effect in Chinese children. J. Exp. Child Psychol. 117, 1-11. https://doi.org/10.1016/j.jecp.2013.08.011 (2014).

59. Campbell, J. I. D. \& Clark, J. M. Cognitive number processing: an encoding-complex perspective. Adv. Psychol. 91(C), 457-491. https://doi.org/10.1016/S0166-4115(08)60894-8 (1992).

60. Dehaene, S., Bossini, S. \& Giraux, P. The mental representation of parity and number magnitude. J. Exp. Psychol. Gen. 122(3), 371-396. https://doi.org/10.1037/0096-3445.122.3.371 (1993).

61. Lemaire, P. \& Fayol, M. When plausibility judgments supersede fact retrieval: the example of the odd-even effect on product verification. Memory Cogn. 23(1), 34-48. https://doi.org/10.3758/BF03210555 (1995).

62. Heubner, L. et al. A mental odd-even continuum account: some numbers may be "more odd" than others and some numbers may be "more even" than others. Front. Psychol. https://doi.org/10.3389/fpsyg.2018.01081 (2018).

63. Fabre, L. \& Lemaire, P. Age-related differences in automatic stimulus-response associations: insights from young and older adults' parity judgments. Psychon. Bull. Rev. 12(6), 1100-1105. https://doi.org/10.3758/BF03206450 (2005).

64. Krueger, L., Hallford, E. W. \& Krueger, L. E. Why $2+2=5$ looks so wrong: on the odd-even rule in sum verification. Memory Cogn. 12, 171-180 (1984).

65. Chochon, F., Cohen, L., van de Moortele, P. F. \& Dehaene, S. Differential contributions of the left and right inferior parietal lobules to number processing. J. Cogn. Neurosci. 11(6), 617-630 (1999).

66. Dehaene, S. et al. Imaging unconscious semantic priming. Nature 395(6702), 597-600. https://doi.org/10.1038/26967 (1998).

67. Pesenti, M., Thioux, M., Seron, X. \& De Volder, A. Neuroanatomical substrates of Arabic number processing, numerical comparison, and simple addition: a PET study. J. Cogn. Neurosci. 12(3), 461-479. https://doi.org/10.1162/089892900562273 (2000).

68. Arsalidou, M. \& Taylor, M. J. Is $2+2=4$ ? Meta-analyses of brain areas needed for numbers and calculations. NeuroImage 54(3), 2382-2393. https://doi.org/10.1016/j.neuroimage.2010.10.009 (2011).

69. Lochy, A. \& Schiltz, C. Lateralized neural responses to letters and digits in first graders. Child Dev. 90(6), 1866-1874. https:// doi.org/10.1111/cdev.13337 (2019)

70. Dehaene, S. \& Cohen, L. Cerebral pathways for calculation: double dissociation between rote verbal and quantitative knowledge of arithmetic. Cortex J. Devot. Study Nervous Syst. Behav. 33(2), 219-250. https://doi.org/10.1016/S0010-9452(08)70002-9 (1997).

71. Takayama, Y., Sugishita, M., Akiguchi, I. \& Kimura, J. Isolated acalculia due to left parietal lesion. Arch. Neurol. 51(3), 286-291. https://doi.org/10.1001/archneur.1994.00540150084021 (1994).

72. Holloway, I. D., Price, G. R. \& Ansari, D. Common and segregated neural pathways for the processing of symbolic and nonsymbolic numerical magnitude: an fMRI study. NeuroImage 49(1), 1006-1017. https://doi.org/10.1016/j.neuroimage.2009.07.071 (2010).

73. Ansari, D., Garcia, N., Lucas, E., Hamon, K. \& Dhital, B. Neural correlates of symbolic number processing in children and adults. NeuroReport 16(16), 1769-1773 (2005).

74. Eger, E., Sterzer, P., Russ, M. O., Giraud, A.-L. \& Kleinschmidt, A. A supramodal number representation in human intraparietal cortex. Neuron 37(4), 719-726. https://doi.org/10.1016/S0896-6273(03)00036-9 (2003).

75. Goffaux, V., Martin, R., Dormal, G., Goebel, R. \& Schiltz, C. Attentional shifts induced by uninformative number symbols modulate neural activity in human occipital cortex. Neuropsychologia 50(14), 3419-3428. https://doi.org/10.1016/j.neuropsych ologia.2012.09.046 (2012)

76. Schuller, A.-M., Hoffmann, D., Goffaux, V. \& Schiltz, C. Shifts of spatial attention cued by irrelevant numbers: electrophysiological evidence from a target discrimination task. J. Cogn. Psychol. 27(4), 442-458. https://doi.org/10.1080/20445911.2014.946419 (2015).

77. Ranzini, M., Dehaene, S., Piazza, M. \& Hubbard, E. M. Neural mechanisms of attentional shifts due to irrelevant spatial and numerical cues. Neuropsychologia https://doi.org/10.1016/j.neuropsychologia.2009.05.011 (2009).

78. Liu-Shuang, J., Norcia, A. M. \& Rossion, B. An objective index of individual face discrimination in the right occipito-temporal cortex by means of fast periodic oddball stimulation. Neuropsychologia 52(1), 57-72. https://doi.org/10.1016/j.neuropsychologi a.2013.10.022 (2014).

79. De Keyser, R., Mouraux, A., Quek, G. L., Torta, D. M. \& Legrain, V. Fast periodic visual stimulation to study tool-selective processing in the human brain. Exp. Brain Res. 236(10), 2751-2763. https://doi.org/10.1007/s00221-018-5331-2 (2018).

80. Lochy, A., Van Belle, G. \& Rossion, B. A robust index of lexical representation in the left occipito-temporal cortex as evidenced by EEG responses to fast periodic visual stimulation. Neuropsychologia 66, 18-31. https://doi.org/10.1016/j.neuropsychologi a.2014.11.007 (2015).

81. Lochy, A., Van Reybroeck, M. \& Rossion, B. Left cortical specialization for visual letter strings predicts rudimentary knowledge of letter-sound association in preschoolers. Proc. Natl. Acad. Sci. 113(30), 8544-8549. https://doi.org/10.1073/pnas.1520366113 (2016).

82. Guillaume, M., Mejias, S., Rossion, B., Dzhelyova, M. \& Schiltz, C. A rapid, objective and implicit measure of visual quantity discrimination. Neuropsychologia 111(February), 180-189. https://doi.org/10.1016/j.neuropsychologia.2018.01.044 (2018)

83. Squires, N. K., Squires, K. C. \& Hillyard, S. A. Two varieties of long-latency positive waves evoked by unpredictable auditory stimuli in man. Electroencephalogr. Clin. Neurophysiol. 38(4), 387-401. https://doi.org/10.1016/0013-4694(75)90263-1 (1975).

84. Norcia, A. M., Appelbaum, L. G., Ales, J. M., Cottereau, B. R. \& Rossion, B. The steady-state visual evoked potential in vision research: a review. J. Vis. 15(6), 4. https://doi.org/10.1167/15.6.4 (2015).

85. Rossion, B. Understanding individual face discrimination by means of fast periodic visual stimulation. Exp. Brain Res. 232(6), 15991621. https://doi.org/10.1007/s00221-014-3934-9 (2014)

86. Dehaene. ,. Varieties of numerical abilities. Cognition 44, 1-42 (1992).

87. Libertus, M. E., Woldorff, M. G. \& Brannon, E. M. Electrophysiological evidence for notation independence in numerical processing. Behav. Brain Funct:: BBF 3(1), 1. https://doi.org/10.1186/1744-9081-3-1 (2007).

88. Temple, E. \& Posner, M. I. Brain mechanisms of quantity are similar in 5-year-old children and adults. Proc. Natl. Acad. Sci. USA https://doi.org/10.1073/pnas.95.13.7836 (1998).

89. Thioux, M., Pesenti, M., Costes, N., De Volder, A. \& Seron, X. Task-independent semantic activation for numbers and animals. Cogn. Brain Res. 24(2), 284-290. https://doi.org/10.1016/j.cogbrainres.2005.02.009 (2005).

90. Hsu, Y.-F. \& Szücs, D. The time course of symbolic number adaptation: oscillatory EEG activity and event-related potential analysis. NeuroImage 59(4), 31033109. https://doi.org/10.1016/j.neuroimage.2011.11.017 (2012).

91. De Vos, T. Test voor het vaststellen van het rekenvaardigheidsniveau der elementaire bewerkingen (automatisering) voor het basis en voortgezet onderwijs: Handleiding. (Berkhout, 1992).

92. Brainard, D. H. The psychophysics toolbox. Spat. Vis. 10(4), 433-436. https://doi.org/10.1163/156856897X00357 (1997).

93. Kleiner, M. et al. What's new in Psychtoolbox-3?. Perception 36, S14. https://doi.org/10.1068/v070821 (2007). 
94. Pelli, D. G. The VideoToolbox software for visual psychophysics: transforming numbers into movies. Spat. Vis. 10(4), 437-442. https://doi.org/10.1163/156856897X00366 (1997).

95. Lochy, A., de Heering, A. \& Rossion, B. The non-linear development of the right hemispheric specialization for human face perception. Neuropsychologia https://doi.org/10.1016/j.neuropsychologia.2017.06.029 (2017).

96. Retter, T. L. \& Rossion, B. Uncovering the neural magnitude and spatio-temporal dynamics of natural image categorization in a fast visual stream. Neuropsychologia 91, 9-28. https://doi.org/10.1016/j.neuropsychologia.2016.07.028 (2016).

97. Boremanse, A., Norcia, A. M. \& Rossion, B. An objective signature for visual binding of face parts in the human brain. J. Vis. 13(11), 66. https://doi.org/10.1167/13.11.6 (2013).

98. Hemptinne, C., Liu-Shuang, J., Yuksel, D. \& Rossion, B. Rapid objective assessment of contrast sensitivity and visual acuity with sweep visual evoked potentials and an extended electrode array. Investig. Opthalmol. Vis. Sci. 59(2), 1144. https://doi.org/10.1167/ iovs.17-23248 (2018).

99. Liu-Shuang, J., Norcia, A. M. \& Rossion, B. An objective index of individual face discrimination in the right occipito-temporal cortex by means of fast periodic oddball stimulation. Neuropsychologia 52, 5772. https://doi.org/10.1016/j.neuropsychologi a.2013.10.022 (2014).

100. Dzhelyova, M. \& Rossion, B. Supra-additive contribution of shape and surface information to individual face discrimination as revealed by fast periodic visual stimulation. J. Vis. 14(14), 15. https://doi.org/10.1167/14.14.15 (2014).

101. Dzhelyova, M. \& Rossion, B. The effect of parametric stimulus size variation on individual face discrimination indexed by fast periodic visual stimulation. BMC Neurosci. https://doi.org/10.1186/1471-2202-15-87 (2014).

102. Cipolotti, L., Butterworth, B. \& Denes, G. A specific deficit for numbers in a case of dense acalculia. Brain 114(6), 2619-2637. https://doi.org/10.1093/brain/114.6.2619 (1991).

\section{Acknowledgements}

The authors would like to thank Aliette Lochy for her advises in data analysis.

\section{Author contributions}

All authors contributed to designing the study; C.S. and A.V.R. directed the project; M.G. created the acquisition software; M.G. and A.P. collected the data; A.P. analyzed the data; A.P. and M.G. wrote the paper with input from A.V.R. and C.S.

\section{Funding}

The current research was funded by the "Luxembourg National Research Fund" (Grant AFR PHD-2015-1/ 9161107).

\section{Competing interests}

The authors declare no competing interests.

\section{Additional information}

Correspondence and requests for materials should be addressed to M.G.

Reprints and permissions information is available at www.nature.com/reprints.

Publisher's note Springer Nature remains neutral with regard to jurisdictional claims in published maps and institutional affiliations.

(c) (i) Open Access This article is licensed under a Creative Commons Attribution 4.0 International License, which permits use, sharing, adaptation, distribution and reproduction in any medium or format, as long as you give appropriate credit to the original author(s) and the source, provide a link to the Creative Commons licence, and indicate if changes were made. The images or other third party material in this article are included in the article's Creative Commons licence, unless indicated otherwise in a credit line to the material. If material is not included in the article's Creative Commons licence and your intended use is not permitted by statutory regulation or exceeds the permitted use, you will need to obtain permission directly from the copyright holder. To view a copy of this licence, visit http://creativecommons.org/licenses/by/4.0/.

(C) The Author(s) 2020 